\title{
Explicit upper bound for an average number of divisors of quadratic polynomials
}

\author{
Kostadinka Lapkova
}

\begin{abstract}
Consider the divisor sum $\sum_{n \leq N} \tau\left(n^{2}+2 b n+c\right)$ for integers $b$ and $c$ which satisfy certain extra conditions. For this average sum we obtain an explicit upper bound, which is close to the optimal. As an application we improve the maximal possible number of $D(-1)$-quadruples.
\end{abstract}

Mathematics Subject Classification (2010). Primary 11N56; Secondary 11D09 .

Keywords. number of divisors, quadratic polynomial, $D(-1)$-quadruples.

\section{Introduction}

Let $\tau(n)$ denote the number of positive divisors of the integer $n$ and $P(x) \in \mathbb{Z}[x]$ be a polynomial. There is a lot of research on estimating average sums of divisors

$$
\sum_{n=1}^{N} \tau(P(n))
$$

One of the ground-laying results was obtained by Erdös [4], who showed that for an irreducible polynomial $P(x) \in \mathbb{Z}[x]$ and for any $N>1$, we have

$$
N \log N \ll_{P} \sum_{n=1}^{N} \tau(P(n)) \ll_{P} N \log N,
$$

where the dependence in the constants can be both on the degree and the coefficients of the polynomial $P(x)$. While for quadratic polynomials there are asymptotic formulas for the sum (1.1), e.g. in works of Hooley [7], McKee [9], [10], and most recently in the paper of Dudek [5], the case $\operatorname{deg} P(x) \geq 3$ is much harder, and no asymptotic formulas for (1.1) are known in this case. A certain progress in this direction was made by Elsholtz and Tao in $\S 7$ of [3].

This work is partially supported by Hungarian Scientific Research Fund (OTKA) grant no. K104183. 
For some applications one needs explicit upper bounds for sum of divisors, rather than asymptotic formulas. Such explicit upper bounds for quadratic polynomials are scarce in the literature, and not always close to the optimal, i.e. with a main term of the same order of magnitude as the main term in the asymptotic formula. For example, for the polynomial $P(n)=n^{2}+1$ one can apply the theorem of McKee [9] and obtain

$$
\sum_{n=1}^{N} \tau\left(n^{2}+1\right)=\frac{3}{\pi} N \log N+\mathcal{O}(N) \sim 0.955 \cdot N \log N .
$$

For this polynomial in Lemma 3.7 of [2] Elsholtz, Filipin and Fujita give the explicit bound

$$
\sum_{n=1}^{N} \tau\left(n^{2}+1\right)<N\left((\log N)^{2}+4 \log N+2\right),
$$

which is clearly larger by a factor of logarithm from the expected growth. This explicit upper bound was improved by Trudgian in [14], but still with a main term of magnitude $N(\log N)^{2}$.

In this note we present an explicit upper bound for (1.1) for a family of quadratic polynomials, which includes the polynomial $P(n)=n^{2}+1$ as well. Our bound will be of the right order of magnitude $N \log N$, as predicted by the asymptotic formulas. The reason for considering only polynomials $P(n)=a n^{2}+b n+c$ with $a=1$ and even integer $b$ is the main role of a certain Dirichlet convolution described in Lemma 2.1 below. Here is our main result.

Theorem 1.1. Let $f(n)=n^{2}+2 b n+c$ for integers $b$ and $c$, such that the discriminant $\delta:=b^{2}-c$ is non-zero and square-free, and $\delta \neq \equiv 1(\bmod 4)$. Assume also that for $n \geq 1$ the function $f(n)$ is positive and non-decreasing. Then for any integer $N \geq 1$ there exist positive constants $C_{1}, C_{2}$ and $C_{3}$, such that

$$
\sum_{n=1}^{N} \tau\left(n^{2}+2 b n+c\right)<C_{1} N \log N+C_{2} N+C_{3} .
$$

Let $A$ be the least positive integer such that $A \geq \max \left(|b|,|c|^{1 / 2}\right)$, let $\xi=$ $\sqrt{1+2|b|+|c|}$ and $\varkappa=g(4|\delta|)$ for $g(q)=\frac{4}{\pi^{2}} \sqrt{q} \log q+0.648 \sqrt{q}$. Then we have

$$
\begin{aligned}
& C_{1}=\frac{12}{\pi^{2}}(\log \varkappa+1), \\
& C_{2}=2\left[\varkappa+(\log \varkappa+1)\left(\frac{6}{\pi^{2}} \log \xi+1.166\right)\right], \\
& C_{3}=2 \varkappa A .
\end{aligned}
$$

The constant $\varkappa$ comes from an effective Pólya-Vinigradov inequality for a real Dirichlet character. We introduce the quantities $\xi=\xi(b, c)$ and 
$A=A(b, c)$, such that always when $n \geq 1$, we have $\sqrt{f(n)} \leq \xi n$ and $\sqrt{f(n)} \leq n+A$.

When we know the precise form of the quadratic polynomial and the corresponding character, we might achieve better upper bounds than the ones provided in Theorem 1.1. This is the case for the polynomial $f(n)=n^{2}+1$.

Theorem 1.2. For any integer $N \geq 1$ we have

$$
\sum_{n=1}^{N} \tau\left(n^{2}+1\right)<\frac{12}{\pi^{2}} N \log N+4.332 \cdot N
$$

We can give an application of Theorem 1.2. Define a $D(n)-m$-tuple for a nonzero integer $n$ and a positive integer $m$ to be a set of $m$ integers such that the product of any two of them increased by $n$ is a perfect square. In the paper of Elsholtz, Filipin and Fujita [2] a crucial role for bounding from above the possible number of $D(-1)$-quadruples plays the inequality (1.3). Plugging the result of Theorem 1.2 in the proof of Theorem 1.3 [2] from the paper of Elsholtz et al. we obtain

Theorem 1.3. There are not more than $4.7 \cdot 10^{58} D(-1)$-quadruples.

This improves the upper bounds $4 \cdot 10^{70}$ from [1], $5 \cdot 10^{60}$ from [2] and $3.01 \cdot 10^{60}$ from [14] for the maximal possible number of $D(-1)$-quadruples, whereas it is conjectured there are none. Note, however, that even if we could supply constants closer to the ones in the asymptotic formula (1.2), we could not achieve essentially useful upper bound for the maximal possible number of $D(-1)$-quadruples without any new ideas. This is due to the method used in the proof of Theorem $1.3[2]$ and the central role of the variable $N \sim 10^{55}$.

\section{Proof of Theorem 1.1}

Since $\delta \neq 0$, the polynomial $f(n)$ is not a full square. It also represents positive non-decreasing function, therefore we can apply the Dirichlet hyperbola method :

$$
\sum_{n \leq N} \tau\left(n^{2}+2 b n+c\right)=\sum_{n \leq N} \sum_{d \mid f(n)} 1=2 \sum_{n \leq N} \sum_{\substack{d \leq \sqrt{f(N)} \\
d \mid f(n)}} 1=2 \sum_{\substack { d \leq \sqrt{f(N)} \\
\begin{subarray}{c}{n \leq N \\
f(n) \equiv 0(d){ d \leq \sqrt { f ( N ) } \\
\begin{subarray} { c } { n \leq N \\
f ( n ) \equiv 0 ( d ) } }\end{subarray}} 1 .
$$

Let

$$
\rho(d):=\#\left\{0 \leq m<d: m^{2}+2 b m+c \equiv 0 \quad(\bmod d)\right\} .
$$

Then for the innermost sum we have

$$
\sum_{\substack{n \leq N \\ f(n) \equiv 0(d)}} 1 \leq\left[\frac{N}{d}\right] \rho(d)+\rho(d) \leq \frac{N}{d} \rho(d)+\rho(d),
$$


so we obtain

$$
\sum_{n \leq N} \tau(f(n)) \leq 2 N \sum_{d \leq \sqrt{f(N)}} \frac{\rho(d)}{d}+2 \sum_{d \leq \sqrt{f(N)}} \rho(d) .
$$

We will bound the sums involving the function $\rho(d)$. For this a crucial role plays the presentation of $\rho(d)$ as a Dirichlet convolution of two other wellunderstood multiplicative functions. More precisely, consider the function $\mu^{2}(n)$, where $\mu$ is the Möbius function, i.e. this is the square-free characteristic function. Also let $\chi(n)$ be the real Dirichlet character given by $\chi(1)=1$ and for $n \geq 1$

$$
\chi(n)= \begin{cases}\left(\frac{\delta}{n}\right) & , \text { if }(n, 2 \delta)=1 \\ 0 & , \text { otherwise }\end{cases}
$$

where $\left(\frac{\delta}{n}\right)$ is the Jacobi symbol.

The following lemma can be considered on the one hand as a generalization of an identity due to Hooley [6], which he shows only for $b=0$. On the other hand, we work on a simplified case, with certain limitations on the discriminant $\delta$. Interestingly, in [7] Hooley claims that with his methods he can give an asymptotic formula for the divisor sum (1.1) for a general quadratic polynomial $P(n)=a n^{2}+b n+c$. Our guess is that he had in mind a similar Dirichlet series presentation as formula (8) in [7], but he never published this argument for the more general case. So, albeit not unexpected, our Lemma has not been published before.

Lemma 2.1. Let $\delta=b^{2}-c$ be square-free and $\delta \not \equiv 1(\bmod 4)$. Given the definitions (2.1) and (2.3), we have the identity

$$
\rho(d)=\sum_{l m=d} \mu^{2}(l) \chi(m) .
$$

Proof. First we notice that $\rho(1)=1, \rho(2)=1$ and $\rho\left(2^{k}\right)=0$ for $k \geq 2$. Indeed, $n^{2}+2 b n+c=(n+b)^{2}-b^{2}+c=(n+b)^{2}-\delta$, so we have

$$
\rho(d)=\#\left\{b \leq x<d+b: x^{2} \equiv \delta \quad(\bmod d)\right\} .
$$

When the integer $\delta$ is odd, the congruence $x^{2} \equiv \delta(\bmod 4)$ has a solution only if $\delta \equiv 1(\bmod 4)$, which is not true by our assumptions. If $\delta$ is even, we do not have solutions of $x^{2} \equiv \delta(\bmod 4)$ because $\delta$ is square-free.

For primes $p>2$ and $(p, \delta)=1$, we have $\rho(p)=\#\left\{0 \leq x<p: x^{2} \equiv \delta(\bmod p)\right\}$ , so $\rho\left(p^{k}\right)=1+\left(\frac{\delta}{p}\right)$ for $k \geq 1$. If $(p, \delta)>1$, clearly $\rho(p)=1$. If $x$ is a solution of $x^{2} \equiv \delta\left(\bmod p^{2}\right)$, then $p$ divides $x$, and $\delta \equiv 0\left(\bmod p^{2}\right)$, which contradicts with $\delta$ being square-free. Therefore $\rho\left(p^{k}\right)=0$ if $k \geq 2$.

For a multiplicative function $\lambda(n)$ we denote the Dirichlet series $D_{\lambda}(s):=$ $\sum_{n=1}^{\infty} \lambda(n) / n^{s}$. By the Chinese Remainder Theorem $\rho(d)$ is multiplicative, but not completely multiplicative. Obviously by definition $(2.1) \rho(d) \leq d$, so 
the Dirichlet series $D_{\rho}(s)$ is absolutely convergent for $\operatorname{Re}(s)>2$. Therefore for $\operatorname{Re}(s)>2$ we can write

$$
\begin{aligned}
D_{\rho}(s) & =\sum_{n \geq 1} \frac{\rho(n)}{n^{s}}=\prod_{p}\left(1+\frac{\rho(p)}{p^{s}}+\frac{\rho\left(p^{2}\right)}{p^{2 s}}+\ldots\right) \\
& =\left(1+\frac{\rho(2)}{2^{s}}\right) \prod_{p>2}\left(1+\frac{\rho(p)}{p^{s}}+\frac{\rho\left(p^{2}\right)}{p^{2 s}}+\ldots\right) \\
& =\left(1+2^{-s}\right) \prod_{\substack{p>2 \\
p \mid \delta}}\left(1+p^{-s}\right) \prod_{\substack{p>2 \\
(p, \delta)=1}}\left(1+\left(1+\left(\frac{\delta}{p}\right)\right)\left(\frac{1}{p^{s}}+\frac{1}{p^{2 s}}+\ldots\right)\right) \\
& =\left(1+2^{-s}\right) \prod_{\substack{p>2 \\
p \mid \delta}}\left(1+p^{-s}\right) \prod_{\substack{p>2 \\
\left(\frac{\delta}{p}\right)=1}}\left(1+2\left(\frac{1}{p^{s}}+\frac{1}{p^{2 s}}+\ldots\right)\right) \\
& =\left(1+2^{-s}\right) \prod_{\substack{p>2 \\
p \mid \delta}}\left(1+p^{-s}\right) \prod_{\substack{p>2 \\
\left(\frac{\delta}{p}\right)=1}}\left(-1+\frac{2}{1-p^{-s}}\right) \\
& =\left(1+2^{-s}\right) \prod_{\substack{p>2 \\
p \mid \delta}}\left(1+p^{-s}\right) \prod_{\substack{p>2 \\
\left(\frac{\delta}{p}\right)=1}} \frac{1+p^{-s}}{1-p^{-s}} .
\end{aligned}
$$

Using definition (2.3) we can write

$$
\prod_{p>2} \frac{1+p^{-s}}{1-\chi(p) p^{-s}}=\prod_{\substack{p>2 \\ p \mid \delta}}\left(1+p^{-s}\right) \prod_{\substack{p>2 \\\left(\frac{\delta}{p}\right)=1}} \frac{1+p^{-s}}{1-p^{-s}} \prod_{\substack{p>2 \\\left(\frac{\delta}{p}\right)=-1}} \frac{1+p^{-s}}{1+p^{-s}}
$$

The third product equals 1 , so we get

$$
\begin{aligned}
D_{\rho}(s) & =\left(1+2^{-s}\right) \prod_{p>2} \frac{1+p^{-s}}{1-\chi(p) p^{-s}}=\prod_{p}\left(1+p^{-s}\right) \prod_{p} \frac{1}{1-\chi(p) p^{-s}} \\
& =D_{\mu^{2}}(s) D_{\chi}(s) .
\end{aligned}
$$

Then the coefficients of the Dirichlet series satisfy the identity

$$
\rho(d)=\sum_{l m=d} \mu^{2}(l) \chi(m) .
$$

For any positive integer $N$ we denote

$$
X(N):=\sum_{1 \leq n \leq N} \chi(n) .
$$

We will need an explicit upper bound for the character sum $|X(N)|$. There are lots of works on such Pólya-Vinegradov inequalities, aiming to reduce the upper bound, e.g. the papers of Qiu [12] and Pomerance [11]. It is a question of taste which one to choose. We will apply the estimate of Qiu since its minor terms are somewhat easier. 
Lemma 2.2. Let $\delta$ be square-free, $\delta \not \equiv 1(\bmod 4)$, and consider the Dirichlet character $\chi$ defined in (2.3). For any $N \geq 1$ we have

$$
\left|\sum_{n=1}^{N} \chi(n)\right|<\varkappa
$$

where $\varkappa=g(4|\delta|)$ and $g(q):=\frac{4}{\pi^{2}} \sqrt{q} \log q+0.648 \sqrt{q}$.

Proof. By the Theorem of Qiu [12] for a primitive Dirichlet character $\chi$ modulo $q$ we have the inequality

$$
\left|\sum_{n=1}^{N} \chi(n)\right|<\frac{4}{\pi^{2}} \sqrt{q} \log q+0.38 \sqrt{q}+0.608 / \sqrt{q}+0.116(N, q)^{2} / q^{\frac{3}{2}} .
$$

Trivially $(N, q)^{2} \leq q^{2}$ and we can further bound from above the latter expression

$$
\left|\sum_{n=1}^{N} \chi(n)\right|<\frac{4}{\pi^{2}} \sqrt{q} \log q+0.496 \sqrt{q}+0.608 / \sqrt{q} .
$$

The expression on the right-hand side suggests to introduce the function $K(x):=\frac{8}{\pi^{2}} x \log x+0.496 x+0.608 / x$. By a simple calculation we can check that for $x \geq 2$ we have $K(x)<\frac{8}{\pi^{2}} x \log x+0.648 x$. Then

$$
\begin{aligned}
\left|\sum_{n=1}^{N} \chi(n)\right| & <K(\sqrt{q})<\frac{8}{\pi^{2}} \sqrt{q} \log (\sqrt{q})+0.648 \sqrt{q} \\
& =\frac{4}{\pi^{2}} \sqrt{q} \log q+0.648 \sqrt{q}=g(q) .
\end{aligned}
$$

Now we return to our character $\chi$ defined in (2.3). We notice that we can write

$$
\chi(n)=\left(\frac{4 \delta}{n}\right),
$$

where $(\dot{)})$ is the Kronecker symbol. Since $\delta \equiv 2,3(\bmod 4)$ is square-free, $4 \delta$ is a fundamental discriminant. Therefore $\chi(n)$ is a primitive character of conductor $q=4|\delta|$. Since $\sqrt{q} \geq 2$, we needed to find a convenient upper bound for $K(x)$ for $x \geq 2$. Now the statement of the Lemma follows from $(2.5)$.

Let $x \geq 1$ be a real number. Using Lemma 2.1 and Lemma 2.2 we get

$$
\begin{aligned}
\sum_{d \leq x} \rho(d) & =\sum_{l m \leq x} \mu^{2}(l) \chi(m)=\sum_{l \leq x} \mu^{2}(l) \sum_{m \leq x / l} \chi(m) \\
& \leq \varkappa \sum_{l \leq x} \mu^{2}(l) \leq \varkappa x .
\end{aligned}
$$


Now returning to $(2.2)$ we see that we need to estimate the sums $\sum_{d \leq x} \rho(d) / d$, for which we use again Lemma 2.1:

$$
\sum_{d \leq x} \frac{\rho(d)}{d}=\sum_{d \leq x} \sum_{l m=d} \frac{\mu^{2}(l) \chi(m)}{l m}=\sum_{l \leq x} \frac{\mu^{2}(l)}{l} \sum_{m \leq x / l} \frac{\chi(m)}{m} .
$$

Consider the sum $\sum_{m \leq x} \chi(m) / m$ for a positive real $x \geq 1$. By Abel's summation formula we have

$$
\Sigma:=\sum_{m \leq x} \frac{\chi(m)}{m}=\frac{X(x)}{x}-\int_{1}^{x} X(u)\left(\frac{1}{u}\right)^{\prime} d u=\frac{X(x)}{x}+\int_{1}^{x} \frac{X(u)}{u^{2}} d u .
$$

If $x \leq \varkappa$, the trivial bound $\left|\sum_{m \leq x} \chi(m)\right| \leq x$ is better than the universal bound provided by Lemma 2.2. Indeed, in that case from (2.8) we obtain

$$
\Sigma \leq \frac{x}{x}+\int_{1}^{x} \frac{u}{u^{2}} d u=1+\log x \leq 1+\log \varkappa .
$$

If $x>\varkappa$, for $(2.8)$ we can write

$$
\begin{aligned}
\Sigma & \leq \frac{\varkappa}{x}+\int_{1}^{\varkappa} \frac{u}{u^{2}} d u+\int_{\varkappa}^{x} \frac{\varkappa}{u^{2}} d u \\
& \leq \frac{\varkappa}{x}+\log \varkappa+\varkappa \int_{\varkappa}^{x} \frac{d u}{u^{2}}=\frac{\varkappa}{x}+\log \varkappa-\frac{\varkappa}{x}+1=\log \varkappa+1 .
\end{aligned}
$$

We conclude that for any $x \geq 1$

$$
\sum_{m \leq x} \frac{\chi(m)}{m} \leq \log \varkappa+1
$$

and then (2.7) transforms into

$$
\sum_{d \leq x} \frac{\rho(d)}{d} \leq(\log \varkappa+1) \sum_{l \leq x} \frac{\mu^{2}(l)}{l} .
$$

For the last sum we apply an explicit upper bound due to Ramaré (Lemma 3.4 in [13]) :

Lemma 2.3. (Ramaré, [13]) Let $x \geq 1$ be a real number. We have

$$
\sum_{n \leq x} \frac{\mu^{2}(n)}{n} \leq \frac{6}{\pi^{2}} \log x+1.166 .
$$

Applying this lemma in (2.10) we get

$$
\sum_{d \leq x} \frac{\rho(d)}{d}<(\log \varkappa+1)\left(\frac{6}{\pi^{2}} \log x+1.166\right) .
$$

We plug the inequalities (2.6) and (2.11), with $x=\sqrt{f(N)}$, into $(2.2)$ :

$$
\begin{aligned}
\sum_{n \leq N} \tau(f(n)) & \leq 2 N(\log \varkappa+1)\left(\frac{6}{\pi^{2}} \log (\sqrt{f(N)})+1.166\right) \\
& +2 \varkappa \sqrt{f(N)} .
\end{aligned}
$$


Now notice that $f(n)=n^{2}+2 b n+c \leq n^{2}+2|b| n+|c| \leq(1+2|b|+|c|) n^{2}$ for $n \geq 1$. Then $\sqrt{f(N)} \leq \xi N$, where $\xi=\xi(b, c)=\sqrt{1+2|b|+|c|}$. Thus $\log (\sqrt{f(N)}) \leq \log \xi+\log N$.

Let $A$ be the least positive integer such that $A \geq \max \left(|b|,|c|^{1 / 2}\right)$. Another way to bound from above $\sqrt{f(N)}$ is by using $f(n)=n^{2}+2 b n+c \leq$ $n^{2}+2|b| n+|c| \leq n^{2}+2 A n+A^{2}=(n+A)^{2}$. Then $\sqrt{f(N)} \leq N+A$.

We apply these two bounds to transform further (2.12).

$$
\begin{aligned}
\sum_{n \leq N} \tau(f(n)) & \leq 2 N(\log \varkappa+1)\left(\frac{6}{\pi^{2}} \log N+\frac{6}{\pi^{2}} \log \xi+1.166\right) \\
& +2 \varkappa(N+A)=\frac{12}{\pi^{2}}(\log \varkappa+1) N \log N \\
& +2\left[\varkappa+(\log \varkappa+1)\left(\frac{6}{\pi^{2}} \log \xi+1.166\right)\right] N+2 \varkappa A \\
& =C_{1} N \log N+C_{2} N+C_{3},
\end{aligned}
$$

with the constants $C_{1}, C_{2}, C_{3}$ defined in (1.4). This proves Theorem 1.1.

\section{Proof of Theorem 1.2}

If we apply Theorem 1.1 for the polynomial $f(n)=n^{2}+1$, we obtain the bound

$$
\sum_{n \leq N} \tau\left(n^{2}+1\right)<2.291 \cdot N \log N+10.026 \cdot N+4.84 .
$$

We can do better if we notice that in (2.3) we actually deal with the odd Dirichlet character modulo 4 :

$$
\chi(n)= \begin{cases}1, & \text { if } n \equiv 1(\bmod 4) ; \\ -1, & \text { if } n \equiv 3(\bmod 4) ; \\ 0, & \text { otherwise }\end{cases}
$$

In this case the character sum $X(N)$ defined in (2.4) can take only values 0 or 1 , so we do not need to use Lemma 2.2. We can replace the expressions $\varkappa$ in (2.6) and $\log \varkappa+1$ in (2.11) simply by 1 . Moreover, the summation in (2.2) over $d \leq \sqrt{N^{2}+1}$ is actually over $d \leq N$. Therefore Theorem 1.2 follows from plugging the estimates (2.6) and (2.11) into (2.2), with $x=N$, and 1 instead of $\varkappa$ and $\log \varkappa+1$.

Remark 3.1. In the estimate (2.6) we used the trivial bound $\sum_{l \leq N} \mu^{2}(l) \leq N$, but we can do slightly better for larger values of $N$. First we can use again Lemma 3.4 (Ramaré, [13]) which says that for $N \geq 1000$ the constant 1.166 from its statement can be substituted by 1.048. Another result of Ramaré (Lemma 3.1, [13]) says that for $N \geq 1700$ we have $\sum_{l \leq N} \mu^{2}(l) \leq 0.62 \cdot N$. After a simple computer check for the cases $1000 \leq N<1700$ we see that this 
holds also for any positive integer in this range. Therefore for any $N \geq 1000$ we have slightly smaller upper bound:

$$
\sum_{n \leq N} \tau\left(n^{2}+1\right) \leq 2 N\left(\frac{6}{\pi^{2}} \log N+1.048\right)+2 \cdot 0.62 \cdot N=\frac{12}{\pi^{2}} N \log N+3.336 \cdot N .
$$

\section{Some examples}

Using McKee's theorem from [9] we can compute numerically the constant $\lambda(\delta)$ from the asymptotic formula

$$
\sum_{n \leq N} \tau(f(n)) \sim \lambda(\delta) N \log N
$$

where $f(n)=n^{2}+2 b n+c$ and $\delta=b^{2}-c<0$. Let $\left(C_{1}, C_{2}, C_{3}\right)$ be the triple of constants from Theorem 1.1, such that

$$
\sum_{n \leq N} \tau(f(n))<C_{1} N \log N+C_{2} N+C_{3} .
$$

With this notation for the polynomial $f(n)=n^{2}+1$ we have $\lambda(-1) \sim 0.955$ and $\left(C_{1}, C_{2}, C_{3}\right) \sim(2.291,10.026,4.84)$, whereas Theorem 1.2 improves this to $\left(C_{1}, C_{2}, C_{3}\right) \sim(1.216,4.332,0)$. In general for large $|\delta|$ we have $C_{1} \sim \log |\delta|$, which is not too far from the coefficient in McKee's formula. By the class number formula one can see that $\lambda(\delta)$ is close to the value of the corresponding Dirichlet $L$-function at 1 .

More examples of the explicit upper bounds for few more polynomials are given in the following table.

\begin{tabular}{|l|l|l|l|}
\hline$f(n)$ & $\delta$ & $\lambda(\delta)$ & $\left(C_{1}, C_{2}, C_{3}\right)$ \\
\hline$n^{2}+1$ & -1 & 0.955 & $(1.216,4.332,0)$ \\
\hline$n^{2}+10 n+27$ & -2 & 1.351 & $(2.97,19.6,50.6)$ \\
\hline$n^{2}+4 n+10$ & -6 & 1.56 & $(3.96,31.9,75.9)$ \\
\hline$n^{2}+52 n+706$ & -30 & 1.395 & $(5.29,84.4,1531.1)$ \\
\hline$n^{2}+10 n-26$ & 51 & - & $(5.71,101.4,480.5)$ \\
\hline
\end{tabular}

The (easy) code for the performed computations can be found in [8]. It can be used to estimate explicitly the divisor sum over any other quadratic polynomial $f(n)$ which satisfies the conditions of Theorem 1.1. 


\section{Acknowledgments}

The author thanks Christian Elsholtz for suggesting this problem. His, András Biró's and the referees' comments on earlier versions of this note are highly appreciated.

\section{References}

[1] N. C. Bonciocat, M. Cipu, M. Mignotte, On D(-1)-quadruples, Publ. Mat. 56 (2012), no. 2, 279-304.

[2] C. Elsholtz, A. Filipin, Y. Fujita, On Diophantine quintuples and D(-1)quadruples, Monatsh. Math. 175 (2014), no. 2, 227-239

[3] C. Elsholtz, T. Tao, Counting the number of solutions to the Erdös-Straus equation on unit fractions, J. Aust. Math. Soc. 94 (2013), no. 1, 50-105

[4] P. Erdős, On the sum $\sum_{k=1}^{x} d(f(k))$, J. London Math. Soc. 27 (1952), 7-15

[5] A. Dudek, On the number of divisors of $n^{2}-1$, Bull. Aust. Math. Soc. (2015), available on CJO2015, doi:10.1017/S0004972715001136

[6] C. Hooley, On the representation of a number as the sum of a square and a product, Math. Z. 69 (1958), 211-227

[7] C. Hooley, On the number of divisors of quadratic polynomials, Acta Math. 110 (1963), 97-114

[8] K. Lapkova, http://www.renyi.hu/ lapkova/papers/explicit_bound_SAGE.txt

[9] J. McKee, On the average number of divisors of quadratic polynomials, Math. Proc. Camb. Philos. Soc. 117 (1995), 389-392

[10] J. McKee, A note on the number of divisors of quadratic polynomials, Sieve methods, exponential sums, and their applications in number theory (Cardiff, 1995), 275-281, London Math. Soc. Lecture Note Ser. 237, Cambridge Univ. Press, Cambridge, 1997

[11] C. Pomerance, Remarks on the Pólya-Vinogradov inequality, Integers 11 (2011), no. $4,531-542$

[12] Z.M. Qiu, An inequality of Vinogradov for character sums (Chinese), Shandong Daxue Xuebao Ziran Kexue Ban 26 (1991), no. 1, 125-128

[13] O. Ramaré, An explicit density estimate for Dirichlet L-series, Math. Comp. 85 (2016), no. 297, 325-356

[14] T. Trudgian, Bounds on the number of Diophantine quintuples, J. Number Theory 157 (2015), 233-249

Kostadinka Lapkova

MTA Alfréd Rényi Institute of Mathematics

Reáltanoda u. 13-15

1053 Budapest

Hungary

e-mail: lapkova.kostadinka@renyi.mta.hu 